EPJ Web of Conferences 116, 10001 (2016)

DOI: $10.1051 /$ epjconf/201611610001

(C) Owned by the authors, published by EDP Sciences, 2016

\title{
AMON: Transition to real-time operations
}

\author{
D.F. Cowen ${ }^{1,2}$, A. Keivani², and G. Tešić \\ ${ }^{1}$ Dept. of Astronomy and Astrophysics, the Pennsylvania State University, Univ. Park, PA 16802, USA \\ ${ }^{2}$ Dept. of Physics, the Pennsylvania State University, Univ. Park, PA 16802, USA
}

\begin{abstract}
The Astrophysical Multimessenger Observatory Network (AMON) will link the world's leading high-energy neutrino, cosmic-ray, gamma-ray and gravitational wave observatories by performing real-time coincidence searches for multimessenger sources from observatories' subthreshold data streams. The resulting coincidences will be distributed to interested parties in the form of electronic alerts for real-time followup observation. We will present the science case, design elements, current and projected partner observatories, status of the AMON project, and an initial AMON-enabled analysis. The prototype of the AMON server has been online since August 2014 and processing archival data. Currently, we are deploying new high-uptime servers and will be ready to start issuing alerts as early as winter 2015/16.
\end{abstract}

\section{Introduction}

Current and future high-energy observatories, representing a joint effort by thousands of scientists, aim to solve longstanding questions about origins of cosmic neutrinos and cosmic rays, as well as the possible existence of gravitational waves. However, the current non-electromagnetic observatories have limited sensitivities for detection of cosmic sources. The number of standalone statistically significant detections by these facilities is expected to be low (e.g., about ten per year for IceCube neutrinos [1-3]). Data streams from these facilities contain a large number of signals that are indistinguishable from background events. These "subthreshold" events from any single observatory cannot be used alone to claim a statistically significant source discovery. However, if subthreshold events from distinct observatories are found in temporal and spatial coincidence, then jointly these events may rise to discovery-level significance. Since many multimessenger sources are expected to be transient, it is also important to detect coincidences in real-time and promptly distribute electronic alerts for follow-up EM observations.

The Astrophysical Multimessenger Observatory Network (AMON) is designed to enable these types of coincidence searches both in real-time and with archival data. It will integrate heterogeneous data from many of the world's leading high-energy (neutrino, cosmic-ray and gamma-ray) and gravitational wave observatories into a single virtual system with combined sensitivity greater than that of any individual facility. More details about the scientific motivation, the design elements and the anticipated discovery potential of AMON are summarized in Ref. [4]. In this proceeding, we present an overview

This is an Open Access article distributed under the terms of the Creative Commons Attribution License 4.0, which permits unrestricted use, distribution, and reproduction in any medium, provided the original work is properly cited. 
Table 1. Triggering and follow-up observatories that have signed AMON Memorandum of Understanding (MoU) or Letters of Collaboration (LoC).

\begin{tabular}{llll}
\hline Observatory & Messenger type & Role & Status \\
\hline ANTARES & neutrinos & triggering & MoU \\
FACT & gamma rays & triggering/follow-up & MoU \\
Fermi LAT & gamma rays & triggering & LoC \\
Fermi GBM & gamma rays & triggering & LoC \\
HAWC & gamma rays & triggering & MoU \\
IceCube & neutrinos & triggering & MoU \\
LIGO & gravitational waves & triggering & LoC \\
LMT & radio waves & follow-up & LoC \\
MASTER & optical photons & follow-up & MoU \\
Pierre Auger & neutrons, neutrinos and gamma rays & triggering & MoU \\
PTF & optical photons & follow-up & LoC \\
Swift BAT & X rays & triggering & MoU \\
Swift XRT & X rays & follow-up & MoU \\
Swift UVOT & UV and optical photons & follow-up & MoU \\
VERITAS & gamma rays & triggering/follow-up & MoU \\
\hline
\end{tabular}

of $A M O N$, its current status including its readiness for the real-time operations, and initial results from an AMON-enabled analysis.

\section{AMON overview and status}

The AMON network consists of triggering and follow-up observatories which have signed the AMON Memorandum of Understanding (MoU) [5], summarized in Table 1. The triggering observatories are sensitive to one or more messengers and typically have large fields of view (FoV) and high duty cycles. Ground based triggering observatories send their candidate events to AMON via direct links in realtime. Events from satellite experiments (e.g., Swift BAT and Fermi LAT) are received by utilizing the Gamma-ray Coordinates Network (GCN) [6]. AMON also uses GCN to distribute its electronic alerts (in the VOEvent format [7]) to the follow-up observatories in real-time. The follow-up observatories are pointing telescopes situated on Earth (e.g., FACT, MASTER, VERITAS, etc.), as well as orbital telescopes (Swift XRT \& UVOT) that respond in near real-time to AMON alerts and perform follow-up observations for electromagnetic counterparts of the multimessenger signal.

Triggering facilities linked by AMON collect heterogeneous data used for detecting multimessenger coincidences. The collected data are not transmitted to AMON in their raw format, but in the higher level "trigger event" form that is specific for each observatory type, in the form of XML files in VOEvent format. Events from each observatory stream contain the following minimal information needed for coincidence searches: a trigger time, an event position with a positional error or probability density function and a false positive rate density in the vicinity of the event $\left(\mathrm{sr}^{-1} \mathrm{~s}^{-1}\right)$. The information from these trigger events is combined statistically by algorithms that search in real-time for temporal and spatial coincidences. For each coincident signal found, an AMON alert is created. Information in the AMON alert includes the best fitted common source position, burst onset time, positional errors, false alarm rate, number of events and type of messengers contributing to a given alert.

The AMON database will store events received in real-time from the AMON member observatories as well as their archival data. In addition, the database stores AMON alerts, observatory configuration models, selection criteria used in alert search algorithms, multiple analysis stream configurations including astrophysical sources, and information from follow-up observations of the AMON alerts. The database underwent successful beta testing by using multiple observatory simulated, and more recently real data (blinded), both using archival data, as well as using data in real-time. Currently, the database holds public data from IceCube-40 and IceCube-59 string configurations, Fermi LAT and Swift BAT 
data. It also stores private archival data from Pierre Auger and ANTARES 2008, and public data from LIGO runs S5 and S6 is in theprocess of being stored.

\subsection{Real-time operations}

In May, 2015, AMON established a connection with GCN [6, 8]. The AMON alert streams at GCN are established as private streams that are initially available only to collaborators. In the future, any of these streams can be made public with the approval of the corresponding observatories whose events participate in that particular alert stream. AMON has already started issuing test alerts (from scrambled data) via GCN to some of its network members. There are two event streams from IceCube that are transmitted to AMON in real-time: the $v_{\mu}$ singlet stream and the high-energy starting event (HESE) stream. The $v_{\mu}$ stream contains subthreshold data at the rate of about $3 \mathrm{mHz}$. The HESE stream will provide events at a much lower rate, but with a much high signal-to-noise ratio. As early as winter 2016, AMON will start issuing real-time alerts via GCN to its collaborators and follow-up facilities.

\section{Neutrino + gamma search}

Here we present the results of archival coincidence analyses using public neutrino data from the 40-string configuration of IceCube (IC40) and contemporaneous public gamma-ray data from Fermi LAT $(\approx 41$ weeks). The IC40 public data only contain neutrinos from the northern hemisphere $(\approx 14,000$ neutrinos $)$. The Fermi LAT data that are being used is selected by imposing additional criteria using Fermi LAT purest (i.e. lowest instrumental background) analysis class (Pass 7-V6 Ultraclean; 4.1 million photon events for the period overlapping IC40). We calculate the angular separation between each photon event and each neutrino and identify all pairs with smaller than $10^{\circ}$ separation. We also apply a temporal cut $(\Delta \mathrm{T})$ of $\pm 50 \mathrm{~s}$ between neutrino and photon arrival times.

To perform the archival coincidence analysis, an unbinned log-likelihood function $(\lambda)$ is used. This metric depends on event arrival direction and its uncertainty as well as a background rejection term and is defined as $\lambda=2 \ln \left(P_{\mathrm{LAT}}\left(\vec{x} \mid \hat{x}_{\gamma}\right) P_{\mathrm{IC}}\left(\vec{x} \mid \hat{x}_{v}\right)\right)-2 \ln \left(B\left(\hat{x}_{\gamma}\right)\right)$, where $\lambda$ is the likelihood parameter, $\hat{x}_{\gamma}$ and $\hat{x}_{v}$ are the arrival direction of the photon and neutrino events, respectively, $\vec{x}$ is the best fit position, $B\left(\hat{x}_{\gamma}\right)$ is the Fermi LAT background rejection term, $P_{\mathrm{LAT}}\left(\vec{x} \mid \hat{x}_{\gamma}\right)$ and $P_{\mathrm{IC}}\left(\vec{x} \mid \hat{x}_{v}\right)$ are the energy dependent point spread functions (PSF) of the LAT and IceCube observatories, respectively. In this metric, large values of $\lambda$ indicate a higher probability correlated pair.

We generate a series of 10,000 scrambled datasets to estimate the null distribution. We scramble time and right ascension of each neutrino event while keeping declination and intrinsic neutrino properties unchanged. Applying the spatial and temporal cuts on all of the $v-\gamma$ pairs, on average $2207 \pm 40$ pairs in each of the 10,000 null datasets are left. Then a $\log$-likelihood $(\lambda)$ value is calculated for each $v-\gamma$ pair from the equation above. Additionally, a series of 10,000 signal tests are created by injecting a limited number of forced coincidences into the null data. Spatial and temporal cuts are applied and $\lambda$ is calculated for each pair.

Unblinding the IC40 data and performing the coincidence analysis with Fermi LAT data result in 2138 coincidences applying the cuts of $\Delta \theta<10^{\circ}$ and $|\Delta \mathrm{t}|<50 \mathrm{~s}$. The AD test statistic on this dataset provides a p-value of about $4 \%$. This p-value is consistent with simulations including the injection of 70-100 signal events. The $\lambda$ distribution plot for unblinded data is presented in Fig. 1 . The null hypothesis and a theoretical signal model with 70 injected signals $\left(N_{\text {sig }}=70\right)$ are also plotted for comparison. The bottom plot in Fig. 1 shows the residuals of data and $N_{\text {sig }}=70$ versus the null hypothesis to better represent the differences between the distributions.

Three different further tests were performed to explore whether real $\operatorname{cosmic} v-\gamma$ pairs are present in the data at $N_{\text {sig }}=70$ level. We perform the tests only on events with high- $\lambda$ values which are more likely to be signal. We checked the mean number of photons in coincidence with each single neutrino, 

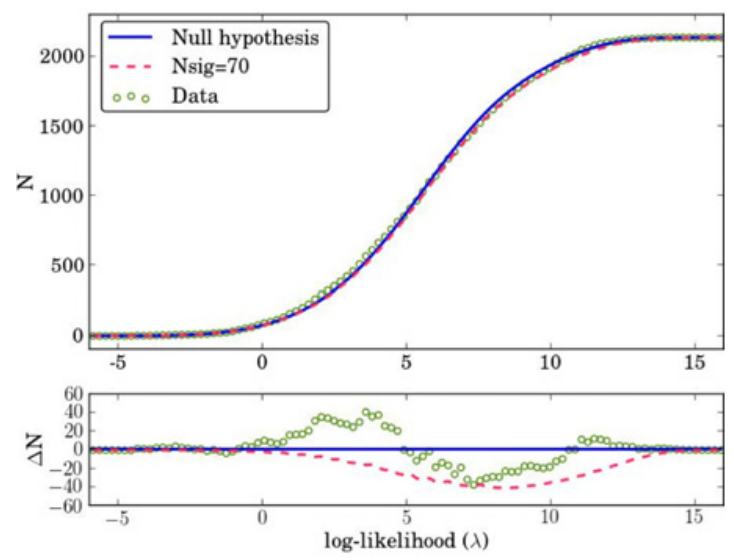

Figure 1. Top: cumulative distribution of log-likelihood values of the IC40 and Fermi LAT coincidences after unblinding the data. A theoretical signal model with 70 injected signals $\left(N_{\text {sig }}=70\right)$ and the null hypothesis are plotted for comparison. All distributions are normalized and multiplied by 2138 , which is the total number of coincidences after unblinding the data. Bottom: The residuals of data and $N_{\text {sig }}=70$ versus the null hypothesis.

the time difference between the photon event and the neutrino, and the clustering of detected pairs. In all cases, we found consistency with background and no evidence for a contribution from $\operatorname{cosmic} v-\gamma$ pairs.

\section{Conclusion}

Multimessenger astroparticle physics is a field that has grown rapidly since the current generation of non-electromagnetic observatories started continuously monitoring large portions of the sky. The AMON cyberinfrastructure enables real-time and archival searches for possible multimessenger sources by conducting joint subthreshold analysis of multimessinger data. AMON has made significant progress in both real-time and archival analysis. In addition to the $\nu+\gamma$ analysis described above, AMON-enabled searches for primordial black hole evaporation [9], $\gamma+($ gravitational wave), and $\nu+($ blazar TeV $\gamma$ ) are underway. AMON will start issuing electronic alerts via GCN in the winter of 2016. Jointly, the AMON partner facilities may probe the high-energy universe via all four forces of nature for the first time in human history.

This research is supported by grants from the U.S. National Science Foundation (003403953) and the Institute for Gravitation and the Cosmos of the Pennsylvania State University.

\section{References}

[1] M.G. Aartsen et al. (IceCube), Science 342, 1242856 (2013), 1311.5238

[2] M.G. Aartsen et al. (IceCube), Phys. Rev. D88, 112008 (2013), 1310.5477

[3] M.G. Aartsen et al. (IceCube), Phys. Rev. Lett. 113, 101101 (2014), 1405.5303

[4] M.W.E. Smith et al., Astropart. Phys. 45, 56 (2013), 1211.5602

[5] The AMON Memorandum of Understanding, http://amon.gravity .psu .edu/mou_aug2012. shtml 
Very Large Volume Neutrino Telescope (VLVnT-2015)

[6] Gamma-ray Coordinates Network (GCN), http://gcn.gsf c.nasa.gov/

[7] International Virtual Observatory Alliance, VOEvent, http://www.ivoa.net/documents/ VOEvent

[8] S. Barthelmy, Private communication

[9] G. Tešić, PoS ICRC2015, 328 (2015) 